\title{
Center-to-limb variation of scattering polarization in molecular solar lines: Observations and modeling
}

\author{
M. Faurobert ${ }^{1}$ and J. Arnaud ${ }^{2}$ \\ ${ }^{1}$ Laboratory for Astrophysics, University of Nice-Sophia-Antipolis, Parc Valrose, 06108 Nice, France \\ 2 UMR 5572, Observatory of Midi-Pyrénées, 14 avenue Édouard Belin, 31400 Toulouse, France
}

Received 7 February 2003 / Accepted 12 September 2003

\begin{abstract}
We present center-to-limb measurements of the scattering polarization observed in molecular lines of $\mathrm{C}_{2}$ and $\mathrm{MgH}$ in the spectral range between 515.7 and $516.1 \mathrm{~nm}$, together with a radiative transfer model for the formation of these lines. The observations were performed in July 2000 with THEMIS inside the south polar limb. We were able to measure the polarization at distances between 1 arcsec and 50 arcsec from the solar limb. The lines appear as very weak absorption features in the intensity spectrum but their linear polarization clearly dominates in the polarization spectrum. We introduce here a simple radiative transfer model which allows to interpret the observed center-to-limb variations of both the intensity and linear polarization. The basic assumption is that molecular lines are formed higher in the photosphere than the continuous photospheric radiation. Molecules are thus illuminated by the polarized continuum photospheric radiation field. We account for a possible Hanle effect due to weak unresolved magnetic fields but we neglect depolarizing collisions. The model depends on four parameters which are determined by fitting both the intensity and polarization in 9 molecular lines of the observed spectral domain. Making use of the differential Hanle effect in the different lines of $\mathrm{C}_{2}$ we show that the $\mathrm{C}_{2}$ lines are affected by the Hanle effect due to a weak unresolved magnetic field. Its mean strength is on the order of 15 Gauss in the upper photosphere and increases to values on the order of 50 Gauss at larger depths. These results are in good agreement with those derived previously (Faurobert et al. 2001) from the linear polarization of the SrI $460.7 \mathrm{~nm}$ line which was observed simultaneously. Such a weak field has almost no effect on the $\mathrm{MgH}$ lines.
\end{abstract}

Key words. techniques: photometric - techniques: spectroscopic - Sun: atmosphere - polarization - scattering

\section{Introduction}

In a previous paper (Faurobert \& Arnaud 2001 hereafter Paper I) we reported on observations of the scattering polarization in molecular emission line observed off-limb, in the low chromosphere with THEMIS. The present paper is devoted to the center-to-limb variation of the linear polarization measured in the same molecular lines when they are observed on the solar disk, as absorption lines. Such observations were first performed by Stenflo et al. (1997) with the ZIMPOL polarimeter at Kitt Peak, but for a smaller range of limb-distances (between 10 arcsec and 20 arcsec from the limb). The linear polarization sharply decreases when the limb distance increases, so that its measurements at large limb distances requires very accurate polarimetry. THEMIS is a telescope which was specially designed for precise measurements of the polarization in the visible solar spectrum (see Arnaud et al. 1998), we present here spectro-polarimetric observations with a polarimetric sensitivity reaching the order of $10^{-5}$. This allows to investigate the center-to-limb variation of the linear polarization in the molecular lines on a larger domain, namely for limb distances

Send offprint requests to: M. Faurobert, e-mail: faurob@unice.fr between 1 arcsec and 40 arcsec. This corresponds to cosines of the heliocentric angle between 0.05 and 0.33 . We also observed linear polarization for larger limb-distances but the accuracy of the measurements is degraded by the presence of residual fringes.

In the second part of this paper we give some details about the observing set-up and reduction procedures and we present the observed intensity and polarization spectra. We show that the accuracy of the polarimetric measurements is mainly limited by the presence of residual fringes in the polarization spectrum.

In the third part we introduce a simple radiative transfer model which allows to interpret the observations. The molecular line forming region is assumed to be a thin layer which is illuminated by the continuum photospheric radiation field, formed below. The incident radiation is polarized due to scattering by free electrons and hydrogen atoms in the photosphere. It is considered as a known quantity, which is derived from an independent radiative transfer calculation of the continuum radiation in the solar photosphere. The incident radiation field is either absorbed or scattered by the molecules. We can consider the radiation field as the superposition of a diffuse radiation 
and of a directly transmitted one. This approach is standard for example in planetary atmosphere modeling (see Chandrasekar 1960). The source function for the diffuse radiation field contains a scattering term and a thermal emission term. We propose an approximate form for the source function based on the assumption that the molecular layer is optically thin. The emergent radiation field is then obtained by an integration of the source function along the line of sight. We obtain analytical expressions for the intensity and polarization as functions of the heliocentric angle. They are very fast to compute even for a large set of model grids.

Let us notice that this model is slightly different from the one we used in PaperI, first because we did not take into account the polarization of the photospheric incident radiation and also because we neglected the attenuation of the transmitted radiation inside the slab. This first approach was valid for emission lines formed by single scattering in a very thin slab.

The present model is more accurate, it relies on four parameters, namely the optical thickness of the molecular layer, the value of the Planck function, the fraction of molecular scattering with respect to absorption processes and the Hanle depolarization coefficient. A parameter study of the model is performed for typical ranges of the parameter values. Then we compare the results to the center-to-limb variations of both the intensity and linear polarization observed in 9 molecular lines inside our spectral domain. We recall that this domain contains $2 \mathrm{MgH}$ lines and 4 triplet lines of $\mathrm{C}_{2}$. The P1 and P2 components of the triplets are blended, they are treated as one single line, whereas the P3 lines are well separated. The most sensitive parameters for the line intensity are the Planck function and the optical thickness of the layer, they can be adjusted to fit the observed center-to-limb variations. The polarization also depends on the product of the scattering and Hanle coefficients. Its value may be determined from the observations. In order to go further and distinguish between scattering polarization and Hanle effect we compare the polarization observed in $\mathrm{C}_{2}$ blended and unblended lines which have different effective Landé factors. We show that their polarization ratio is different from what is expected in the absence of Hanle effect. As all the triplet lines of $\mathrm{C}_{2}$ have the same radiative transition rate and probably the same sensitivity to depolarizing collisions as well but a different sensitivity to the Hanle effect, the polarization ratio of blended and unblended lines depends on the magnetic strength only. This is an ideal case for implementing a diagnostics procedure based on the so-called differential Hanle effect (see Stenflo et al. 1998).

The Landé factors and life-times of the relevent molecular levels are given in Table 2 (from Berdyugina 2003), from these values we derive that the sensitivity domains to the Hanle effect are for magnetic fields on the order of 28 Gauss, 56 Gauss and of 160 Gauss respectively for the $P 3$ lines of $\mathrm{C}_{2}$, for the blended $P 1$ and $P 2$ lines and for the $\mathrm{MgH}$ lines. By measuring the differential Hanle effect we derive the depolarization factor for the $\mathrm{C}_{2}$ lines, and the mean strength of the weak unresolved magnetic field. We obtain values between 0 . and 15 Gauss in the upper photosphere, at limb-distances between 1 arcsec and 20 arcsec, and larger values on the order of 45 Gauss at limbdistances of 40 arcsec corresponding to deeper regions in the photosphere. This is in quite good agreement with the values between 20 Gauss and 30 Gauss derived previously from the linear polarization of the SrI line at $460.7 \mathrm{~nm}$ (Faurobert et al. 2001) which was observed simultaneously.

Such a weak field has almost no effect on the polarization in the $\mathrm{MgH}$ lines.

\section{Observations}

The observations were made for slit positions very close to the solar south pole under good seeing conditions. The polarization analysis package of THEMIS (cf. Paletou \& Molodij 2001) consists in two identical Fichou achromatic quarter-wave elements, each made up of two plates in quartz and in $\mathrm{MgF}_{2}$, followed by a calcite beam splitter. Two beams of orthogonal polarization exit the polarimeter and go through the spectrometer for spectral analysis. They were recorded on one single CCD. High precision spectropolarimetry can be achieved with such a system by applying the beam exchange technique (Semel 1994; Donati et al. 1990). The beam exchange was at the time of the observations only possible for one of the linear polarization Stokes parameter. We performed it for the Stokes parameter $Q$. This implied to orientate the slit at $45^{\circ}$ from the solar limb direction. The parameter $Q$ is defined in a standard way, with respect to a system of axis parallel and perpendicular to the solar limb (see next section). In this reference system, for symmetry reasons, both $U$ and $V$ are equal to zero in regions where there is no net magnetic flux. We could check that in the regions where we observed, $U$ and $V$ are indeed below our detection limit, less than one order of magnitude smaller than $Q$. Let us notice that we always observed linear polarization parallel to the solar limb, which corresponds to negative values of $Q$, according to the standard definition of Chandrasekhar. In this paper we thus show $-Q$ in all the figures, this allows a more clear presentation of the results.

For observations up to 20 arcsec from the solar limb, we kept the limb inside the slit field of view (Fig. 1). and with the slit direction at $45^{\circ}$ of the solar limb. The slit width is $0.5 \operatorname{arcsec}$ and the pixel size is 0.28 arcsec in the direction perpendicular to the limb. The spectral resolution is of $1.6 \mathrm{pm}$ per pixel. The solar limb position is defined as the inflexion point of the limb darkening curve.

The observation procedure we used, together with the reduction technique that we applied, are described in details in Faurobert et al. (2001). The exposure time was of $2 \mathrm{~s}$ and we added 300 polarization sequences to accumulate enough photons to reach an adequate polarimetric sensitivity. A polarization sequence consists, for each beam, in four images, one for each of the four different orientations of the quarter-waves plates we used (for each image, one gets $I+$ or - an other Stokes parameter, \pm means that the sign is + for the first beam and - for the second beam, $\mp$ meaning the reverse):

$$
\begin{aligned}
& S_{0}=I \pm Q, \quad S_{1}=I \mp Q, \quad S_{2}=I \pm U, \\
& S_{3}=I \mp V .
\end{aligned}
$$

Actually, the Fichou elements are not perfectly achromatic so not exactly quarter-wave at $5160 \AA$. For a retardation of $\Phi$ in 

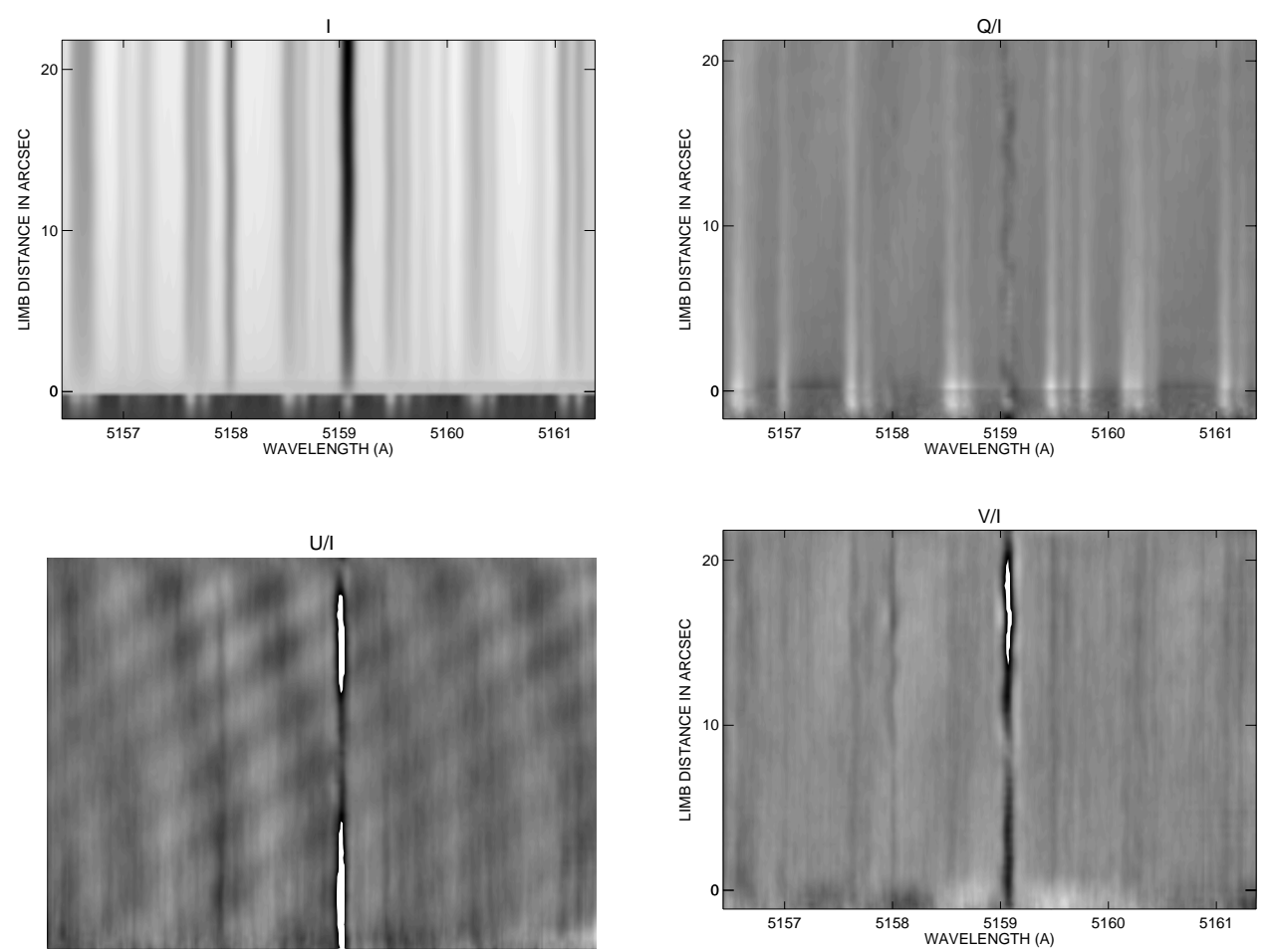

Fig. 1. From left to right and top to bottom: observed $I,-Q / I, U / I, V / I$ spectral images of the molecular lines domain. $I$ does appear in emission above the solar limb where $Q / I$ is also visible. $Q / I$ decreases in absolute value with the distance from the limb and is, in the line core, of the order of $10^{-3}$ at 5 arcsec inside the Sun and of $5 \times 10^{-4}$ at 20 arcsec. On $U / I$ and $V / I$ spectral images are only visible, mostly below the $5 \times 10^{-4}$ level, fringes and line intensity residuals.

place of $90^{\circ}$ the intensities obtained for the four images of a polarization sequence, are now related to $I, Q, U$ and $V$ by:

$S_{0}=I \pm Q$,

$S_{1}=I \mp Q\left(2 \sin ^{2} \Phi-1\right) \mp 2 V \sin \Phi \cos \Phi$,

$S_{2}=I \pm Q \cos \Phi \pm U \sin ^{2} \Phi \mp V \sin \Phi \cos \Phi$,

$S_{3}=I \pm Q \cos \Phi \mp V \sin \Phi$.

$\Phi$ being known, the four Stokes parameters can be easily computed for each of the two beams, from the simple linear combinations of the four measurements of a sequence which can be deduced - for instance by inverting a $4 \times 4$ matrix - from the above linear relations. Adding all the polarization sequences and combining the results obtained for each of the two beams, we end up with Stokes parameters free of beam differences and seeing effects. At $5160 \AA, \Phi=95^{\circ}$. For our observations $U$ and $V$ are at least an order of magnitude lower than $Q$. In this case, $Q$ is almost not sensitive to this departure of the retarders from quarter-wave.

A polarimetric sensitivity of $0.001 \%$ to $0.002 \%$ can be achieved for such polarization sequences, with averaging over a small interval of $\mu(=\cos \theta), \theta$ is the angle between the propagation direction and the local solar radius.

Figure 2 shows the intensity spectrum, normalized by the intensity $I_{\mathrm{c}}$ observed in the continuum at disk center, together with $-Q / I$ at $\mu=0.33$.

The flat-field images have been built up from scans made while the telescope was moving in a way to have the solar image describing a pseudo-ellipse centered near the solar disc, in a location chosen to avoid active regions passing across the field of view. The flat-field scans images were stacked for each of the four retarder positions. The four resulting images were destretched to have wavelength variations along the detector rows and spatial variations along the columns. They were then flattened in the usual way, dividing each image by its average row in order to remove the spectral features. "Channel fringes" (cf. Semel 2003) do form inside the analysis package. They can be removed to a large extend, not perfecty, by flat-fielding, as explained in Faurobert et al. (2001).

The Themis polarization analysis package is installed at the first focus of this Ritchey-Chretien telescope, before any folding mirror. At this level, instrumental polarization may only occurs from birefringency inside the closing windows of the helium filled telescope tube. There is actually no evidence of such effects which, anyway, can not produce cross-talk between $I$ and the other Stokes parameters. For this reason, Themis is the only present time large solar telescope where the zero polarization level should be obtained free of instrumental effects. This may allow continuum polarization measurements which are very important for second solar spectrum observations where lines scattering polarization is of the same order as continuum polarization. However, for the observations reported here, continuum polarization is not very well measured due to fringe residuals which, more generally, limit the polarimetric precision. It is why we still use model values for continuum polarization. Figure 3 presents our continuum polarization measurements, compared to the model polarization, both are very consistent (notice the oscillations of the 


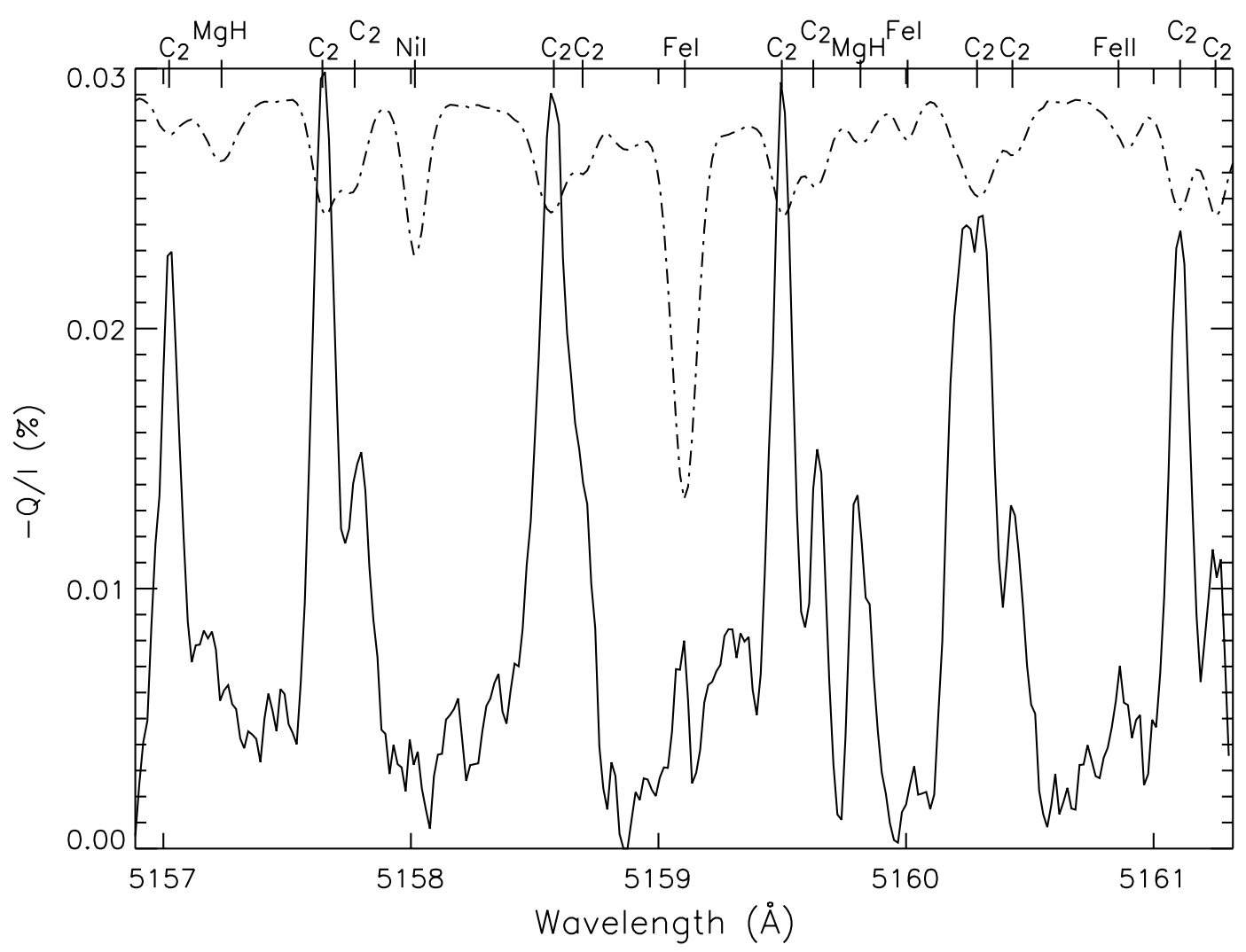

Fig. 2. $I$, normalized by the intensity $I_{c}$ in the continuum at disk center and multiplied by $5 \times 10^{-4}$, (dashed-dotted line) and $-Q / I$ at $\mu=0.33$. Fringe residuals are present, at a level of $5 \times 10^{-5}$ peak to valley, the noise level for $Q / I$ is about $1 \times 10^{-5}$.

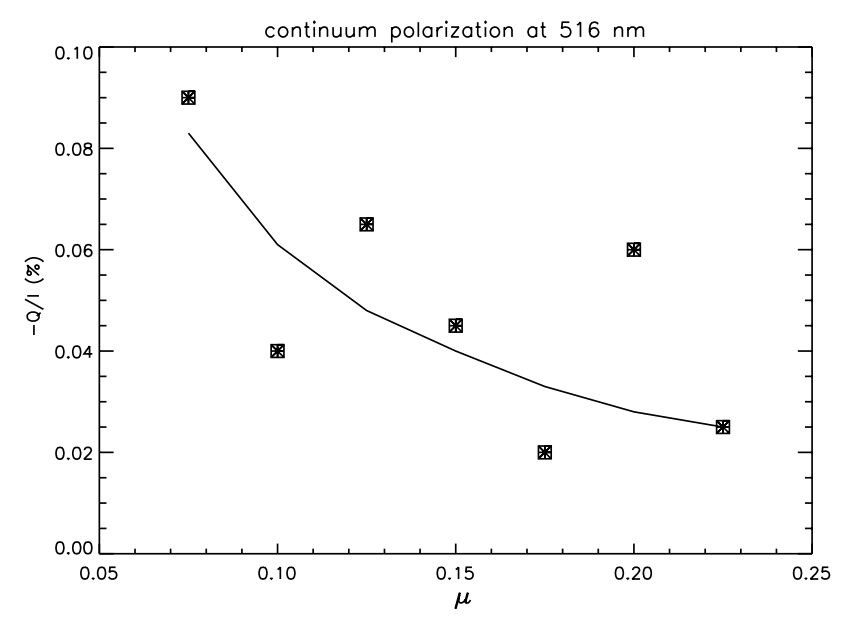

Fig. 3. Continuum polarization measured for several values of $\mu$, and (full line) the polarization obtained from the solar atmosphere model. Measurements validate the model even if scattered by $4 \times 10^{-4}$ peak to valley amplitude fringe residuals.

polarization with respect to the model curve, due to fringe residuals). Continuous radiation polarization was measured by Leroy (1972) and Mickey \& Orrall (1974) but with broad-band filters. Wiehr (1975) performed spectro-polarimetric measurements but for different spectral domains and with a lower accuracy. Recently Wiehr \& Bianda (2003) measured the linear scattering polarization in the blue domain with interferometric filters quite close to the solar limb.

\section{Radiative transfer modeling}

\subsection{Diffuse radiation field}

Up to now very few theoretical studies have been devoted to the formation of polarized molecular lines. Mohan Rao \& Rangarajan (1999) proposed an interpretation of the linear polarization observed by Stenflo \& Keller (1997) in MgH absorption lines at $516.5933 \mathrm{~nm}$ and $516.8147 \mathrm{~nm}$, close to the solar limb and a simple single scattering model was used to interpret the emission line observations in Paper I.

In the present model, the thin molecular layer is illuminated by the incident continuous radiation emitted by the photosphere. Due to limb-darkening, the photospheric radiation field is anisotropic and polarized. Inside the slab, the incident radiation is scattered by molecular dipole-type transitions.

In the spectral domain where we observed, the molecular lines of $C_{2}$ and $M g H$ are due to electronic transitions $A^{3} \Pi_{g}$ $X^{3} \Pi_{u}$ and $A^{2} \Pi_{3 / 2,1 / 2}-X^{2} \Sigma$, respectively, in the vibration band $(0,0)$. The $C_{2}$ lines belong to the $\mathrm{P}$ branch. In our observations they appear as doublets; the "blue" line of the doublets correspond to $P 1$ and $P 2$ components, which are blended, and the "red" component belongs to the $P 3$ branch. The identification and rotational quantum numbers for all the molecular lines of our spectral domain are taken from Pierce (1968) and given in Table 2 .

As the molecular absorption lines are very weak (see Fig. 1) we assume that the molecular layer is optically thin. 
We account for a possible depolarizing Hanle effect of weak and unresolved magnetic fields.

The incident radiation field is symmetrical with respect to the radial axis and varies with respect to the angle $\theta$ between its propagation direction and the local solar radius. It does not dependent on frequency in the spectral interval of interest. Its specific intensity and polarization are denoted by $I_{\text {inc }}(\mu)$ and $Q_{\text {inc }}(\mu)$, respectively, where $\mu$ is the cosine of $\theta$.

Inside the slab, the radiation field is either scattered by molecules or absorbed by the slab material. We denote by $k_{\mathrm{sc}}$ the scattering opacity and by $k_{\mathrm{a}}$ the opacity due to absorption processes. The absorption term is balanced by a thermal emission term which is proportional to the Planck function. We introduce the optical thickness $\tau$ such that $\mathrm{d} \tau=-\left(k_{\mathrm{a}}+k_{\mathrm{sc}}\right) \mathrm{d} z$ and the Stokes vector for the linearly polarized radiation field $\boldsymbol{I}=(I, Q)^{\dagger}$. We define here the Stokes parameters $I$ and $Q$, as in Chandrasekhar (1960); $I$ is the specific intensity of the radiation field, and $Q=I_{1}-I_{\mathrm{r}}$, where $I_{1}$ and $I_{\mathrm{r}}$ denote the components which are respectively perpendicular and parallel to the limb. According to this definition, negative values of $Q$ correspond to a linear polarization parallel to the limb. The polarized transfer equation is written as

$$
\begin{aligned}
\mu \frac{\mathrm{d} \boldsymbol{I}(\tau, \mu)}{\mathrm{d} \tau}= & \boldsymbol{I}(\tau, \mu)-\frac{k_{\mathrm{sc}}}{k_{\mathrm{sc}}+k_{\mathrm{a}}} \int_{-1}^{1} \frac{\mathrm{d} \mu^{\prime}}{2} \hat{P}\left(\mu, \mu^{\prime}\right) \boldsymbol{I}\left(\tau, \mu^{\prime}\right) \\
& -\frac{k_{\mathrm{a}}}{k_{\mathrm{sc}}+k_{\mathrm{a}}} \boldsymbol{B}_{\mathrm{th}} .
\end{aligned}
$$

In the following we shall use the notation $c_{\mathrm{sc}}=k_{\mathrm{sc}} /\left(k_{\mathrm{sc}}+k_{\mathrm{a}}\right)$, the vector $\boldsymbol{B}_{\mathrm{th}}=\left(B_{\mathrm{th}}, 0\right)^{\dagger}$ where $B_{\mathrm{th}}$ is the Planck function. The notation ${ }^{\dagger}$ denotes the transposed vector. In the scattering integral $\hat{P}\left(\mu, \mu^{\prime}\right)$ is the scattering phase matrix, $\mu$ and $\mu^{\prime}$ refer respectively to the propagation directions of the re-emitted and of the incident radiation fields. Here, we do not take into account any frequency redistribution mechanism. This is consistent with the approximations that we shall introduce in the following, showing that the source function depends mainly on the incident radiation which is frequency independent (see Eqs. (14) and (15)).

The general form of the scattering phase matrix for dipolar electric transitions in the presence of Hanle effect of a weak unresolved magnetic field is

$\hat{P}\left(\mu, \mu^{\prime}\right)=\hat{A}(\mu) \hat{A}^{\dagger}\left(\mu^{\prime}\right)$,

with

$\hat{A}(\mu)=\left(\begin{array}{cc}1 & \sqrt{\frac{W_{2} W_{B}}{8}}\left(1-3 \mu^{2}\right) \\ 0 & 3 \sqrt{\frac{W_{2} W_{B}}{8}}\left(1-\mu^{2}\right)\end{array}\right)$.

The coefficient $W_{2}$ is the intrinsic polarizability of the line. Berdyugina et al. (2002) showed that $W_{2}=0.4$ for the $\mathrm{MgH}$ lines in our domain and that $W_{2}=0.1$ for the $C_{2}$ lines we are considering here. The factor $W_{B}$ is the Hanle depolarizing factor. For the special case of a microturbulent magnetic field with a single-valued field strength and an isotropic angular distribution (Stenflo 1982) it is related to the Hanle coefficient $\gamma_{\mathrm{H}}$ by

$W_{B}=1-0.4\left(\frac{\gamma_{\mathrm{H}}^{2}}{1+\gamma_{\mathrm{H}}^{2}}+\frac{4 \gamma_{\mathrm{H}}^{2}}{1+4 \gamma_{\mathrm{H}}^{2}}\right)$, where

$\gamma_{\mathrm{H}}=0.88 g_{J} \frac{B}{\Gamma_{\mathrm{R}}+D^{(2)}}$,

is the Hanle parameter, which depends on the Landé factor of the line $\left(g_{J}\right)$, the mean magnetic intensity $(B)$, here given in Gauss, the line transition rate $\left(\Gamma_{\mathrm{R}}\right)$ and the depolarizing collision rate $\left(D^{(2)}\right)$, here both given in units of $10^{7} \mathrm{~s}^{-1}$. When the magnetic strength increases from zero to infinity the Hanle depolarizing factor $W_{B}$ decreases from 1 to 0.2 .

The transfer Eq. (1) has to be solved with the boundary condition

$\boldsymbol{I}(T, \mu)=\boldsymbol{I}_{\mathrm{inc}}(\mu), \quad$ for $\quad \mu>0$,

where $\boldsymbol{I}_{\text {inc }}$ is the photospheric incident radiation field, it is defined for positive values of $\mu$ only.

Let us introduce the diffuse radiation field $\boldsymbol{I}_{\mathrm{d}}(\tau, \mu)$ such that

$\left.\boldsymbol{I}(\tau, \mu)=\boldsymbol{I}_{\mathrm{d}}(\tau, \mu)+\boldsymbol{I}_{\mathrm{inc}}(\mu) \exp (-T-\tau) / \mu\right)$,

$T$ denotes the optical thickness of the molecular layer, the second term is the transmitted radiation field at depth $\tau$ in the direction $\mu$. Substituting this expression in the transfer equation for $\boldsymbol{I}$ we find that the diffuse radiation field obeys the transfer equation

$\mu \frac{\mathrm{d} \boldsymbol{I}_{\mathrm{d}}(\tau, \mu)}{\mathrm{d} \tau}=\boldsymbol{I}_{\mathrm{d}}(\tau, \mu)-\boldsymbol{S}_{\mathrm{d}}(\tau, \mu)$,

with

$$
\begin{aligned}
\boldsymbol{S}_{\mathrm{d}}(\tau, \mu)= & c_{\mathrm{sc}} \int_{-1}^{1} \frac{\mathrm{d} \mu^{\prime}}{2} \hat{P}\left(\mu, \mu^{\prime}\right) \boldsymbol{I}_{\mathrm{d}}\left(\tau, \mu^{\prime}\right) \\
& +c_{\mathrm{sc}} \int_{0}^{1} \frac{\mathrm{d} \mu^{\prime}}{2} \hat{P}\left(\mu, \mu^{\prime}\right) \boldsymbol{I}_{\mathrm{inc}}\left(\mu^{\prime}\right) \exp \left(-(T-\tau) / \mu^{\prime}\right) \\
& +\left(1-c_{\mathrm{sc}}\right) \boldsymbol{B}_{\mathrm{th}}
\end{aligned}
$$

The boundary condition for Eq. (8) is

$\boldsymbol{I}_{\mathrm{d}}(T, \mu)=0, \quad$ for $\quad \mu>0$.

The source function for the diffuse radiation field is formed by the sum of three terms, namely the scattering integral of the diffuse field, the scattering integral of the transmitted field and a thermal creation term which does not give rise to polarization.

The formal solution of Eq. (8) for the emergent radiation field yields

$\boldsymbol{I}(0, \mu)=\int_{0}^{T} \boldsymbol{S}_{\mathrm{d}}(\tau, \mu) \exp (-\tau / \mu) \frac{\mathrm{d} \tau}{\mu}+\boldsymbol{I}_{\text {inc }}(\mu) \exp (-T / \mu)$.

We now introduce two approximations, which are valid for optically thin slabs. First, we assume that the source function $S_{\mathrm{d}}$ is independent of the optical depth in the slab, the emergent radiation field may thus be written as

$\boldsymbol{I}(0, \mu)=\boldsymbol{S}_{\mathrm{d}}(\mu)(1-\exp (-T / \mu))+\boldsymbol{I}_{\text {inc }}(\mu) \exp (-T / \mu)$,

where $\boldsymbol{S}_{\mathrm{d}}(\mu)$ is computed at slab center.

We also assume that the scattering term of the diffuse radiation field is negligible with respect to the scattering term of 
the transmitted radiation. The diffuse source function is thus given by

$$
\begin{aligned}
\boldsymbol{S}_{\mathrm{d}}(\mu)= & c_{\mathrm{sc}} \int_{0}^{1} \frac{\mathrm{d} \mu^{\prime}}{2} \hat{P}\left(\mu, \mu^{\prime}\right) \boldsymbol{I}_{\mathrm{inc}}\left(\mu^{\prime}\right) \exp \left(-\left(T / 2 \mu^{\prime}\right)\right. \\
& +\left(1-c_{\mathrm{sc}}\right) \boldsymbol{B}_{\mathrm{th}} .
\end{aligned}
$$

Making use of the factorization of the scattering phase matrix given in Eq. (2) we derive the explicit expressions of the two components of $S_{\mathrm{d}}$,

$$
\begin{aligned}
S_{I}(\mu)= & c_{\mathrm{sc}} \int_{0}^{1} \frac{\mathrm{d} \mu^{\prime}}{2} I_{\mathrm{inc}}\left(\mu^{\prime}\right) \exp \left(-T / 2 \mu^{\prime}\right) \\
& +c_{\mathrm{sc}} \frac{W_{2} W_{B}}{8}\left(1-3 \mu^{2}\right) I_{1}(T) \\
& +\left(1-c_{\mathrm{sc}}\right) B_{\mathrm{th}},
\end{aligned}
$$

and

$\boldsymbol{S}_{Q}(\mu)=c_{\mathrm{sc}} \frac{3 W_{2} W_{B}}{8}\left(1-\mu^{2}\right) I_{1}(T)$,

with

$$
\begin{aligned}
I_{1}(T)= & \int_{0}^{1} \frac{\mathrm{d} \mu^{\prime}}{2} \exp \left(-T / 2 \mu^{\prime}\right) \\
& \times\left[\left(1-3 \mu^{\prime 2}\right) I_{\text {inc }}\left(\mu^{\prime}\right)+3\left(1-\mu^{\prime 2}\right) Q_{\text {inc }}\left(\mu^{\prime}\right)\right] .
\end{aligned}
$$

The integrals are restricted to positive values of $\mu$ because the incident radiation propagates outwards. Let us remark here that these approximations may be considered as a generalization of the so-called "last scattering approximation" (Stenflo 1994) which allows to take into account consistently the coupling of the line with the continuum, avoiding the ad-hoc definition of any "effective" $W_{2}$ coefficient.

\subsection{Computation of grids of models}

We first give in Table 1 the limb-darkening $I_{\text {inc }}(\mu) / I_{\text {inc }}(1)$ and polarization degree $-Q_{\text {inc }}(\mu) / I_{\text {inc }}(\mu)$ for the incident photospheric radiation field at $\lambda=5160 \AA$, (the calculations are described in Paper I).

The analytical expression (12) for the radiation field, with the approximations (14) and (15) for the source function, is very easily computed for a set of parameter values for $T, B_{\mathrm{th}}$, $c_{\mathrm{sc}}$ and $W_{B}$. The Planck function values are taken between $0.4 \times 10^{-5}$ and $1.3 \times 10^{-5}$, which corresponds to the temperature range from the middle to the upper photosphere. The optical thickness varies between 0.01 to 0.15 by step of 0.01 , the scattering coefficient between 0.02 to 0.20 by step of 0.01 and the Hanle depolarizing coefficient from 1 to 0.2 by step of 0.1 .

Figures 4 and 5 show respectively the center-to-limb variations of the intensity and of the polarization degree $-Q / I$ obtained from the model for lines with an intrinsic polarizability $W_{2}=0.4$, together with the observed values in the $2 \mathrm{MgH}$ lines of our spectral domain. For the $\mathrm{C}_{2}$ lines we distinguish the blended and unblended lines which have different optical depths and different polarization rates. Figures 6 and 7 are devoted to the $\mathrm{C}_{2}$ blended lines at $515.76 \mathrm{~nm}, 515.85 \mathrm{~nm}$
Table 1. Center-to-limb variations of the continuum intensity and polarization degree at $\lambda=5160$. $\AA$. Results of a radiative transfer calculation for the quiet Sun.

\begin{tabular}{ccc}
\hline \hline$\mu$ & $I_{\text {inc }}(\mu) / I_{\text {inc }}(\mu=1)(\%)$ & $-Q_{\text {inc }} / I_{\text {inc }}(\%)$ \\
\hline 0.05 & 31.8 & 0.083 \\
0.075 & 34.6 & 0.061 \\
0.10 & 37.1 & 0.048 \\
0.125 & 39.5 & 0.040 \\
0.15 & 41.9 & 0.033 \\
0.173 & 43.9 & 0.029 \\
0.20 & 46.3 & 0.025 \\
0.33 & 56.8 & 0.013 \\
0.41 & 62.0 & 0.010 \\
0.50 & 69.1 & 0.007 \\
0.60 & 75.8 & 0.004 \\
0.70 & 82.2 & 0.003 \\
0.80 & 88.4 & 0.001 \\
1.0 & 1. & 0. \\
\hline
\end{tabular}

$515.95 \mathrm{~nm}$ and $516.10 \mathrm{~nm}$, and Figs. 8 and 9 to the $\mathrm{C}_{2}$ unblended lines at $515.77 \mathrm{~nm}$ and $515.87 \mathrm{~nm}$. On each figure the 4 panels correspond to the grid of models obtained when one of the four parameters varies, the 3 others being set to the values which give the best fit to the observed quantities. The optimal choice of parameter values is given in Table 2 .

We see in Figs. 4-9 that the line intensity depends both on the value of the Planck function and on the optical thickness, but is not sensitive to the scattering coefficient and to the Hanle depolarizing coefficient. This is due to the relatively small values of the scattering coefficient that we must consider in order to account for the observed polarization rates. The fitting of the line intensity allows to determine the Planck function and optical thickness. We notice that the Planck function is the same for all the lines of our spectral domain, i.e. $B_{\mathrm{th}}=0.9 \times 10^{-5}$, this corresponds to the quiet sun value at the altitude $z=300 \mathrm{~km}$, in the photosphere. As expected, the optical thickness of the blended lines of $C_{2}$ is twice that of the unblended lines. Once $T$ and $B_{\text {th }}$ are determined, we adjust the product $c_{s c} W_{B}$ in order to fit the observed polarization rates. The fitting is indeed possible with one single value of $c_{\mathrm{sc}} W_{B}$ for the $\mathrm{MgH}$ lines, whereas for the $\mathrm{C}_{2}$ lines we have to consider depth-variations of this product. In Table 2 we give an average value, valid for $\mu<0.2$.

Let us now explain how we can determine separately $c_{\mathrm{sc}}$ and $W_{B}$. We can first notice that the scattering coefficients $c_{\mathrm{sc}}$ are the same for the blended and unblended lines, because both the absorption and the scattering opacities are multiplied by a factor of two for the blended lines as compared to the unblended ones. However the Hanle depolarization factors in the presence of a weak unresolved magnetic field are different in both types of lines. The reason is that their effective Landé factors are differents. The Landé factors of the molecular energy levels are given in Table 2, we see that the P2 lines are almost not sensitive to magnetic fields, and that $\mathrm{P} 1$ and $\mathrm{P} 3$ lines have almost the same Landé factors, in absolute value. The absolute value of the effective Lande factor for the blended P1 and P2 lines is thus half the value of the P3 ones. We recall that the 


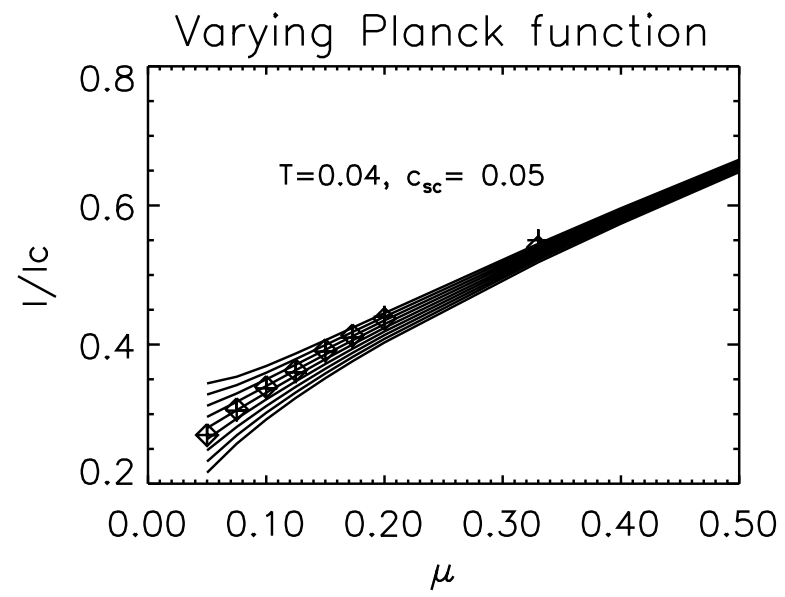

(a)

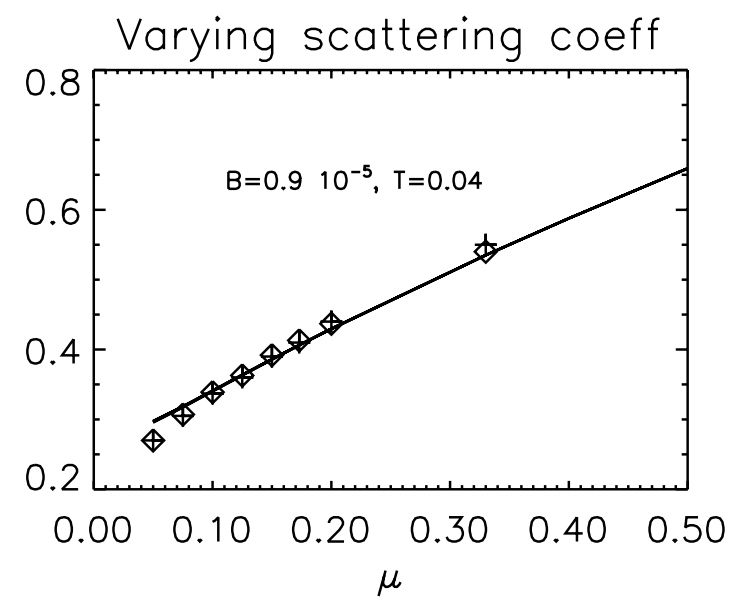

(c)

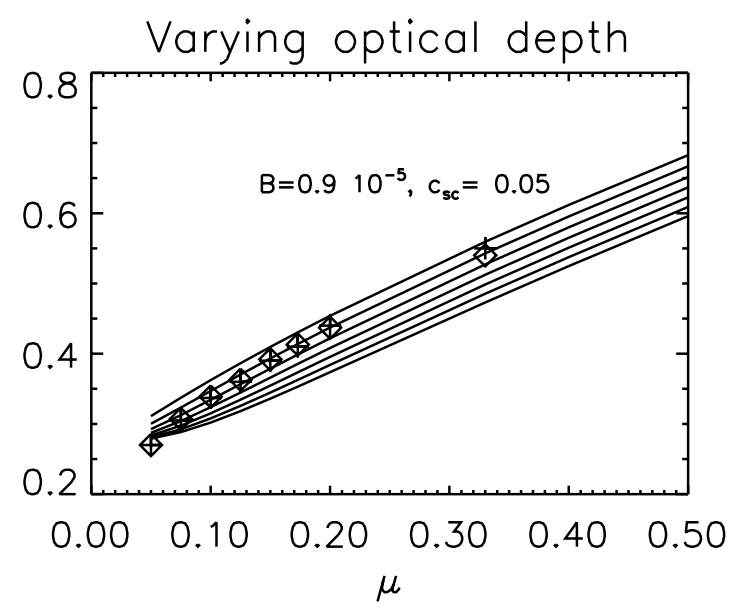

(b)

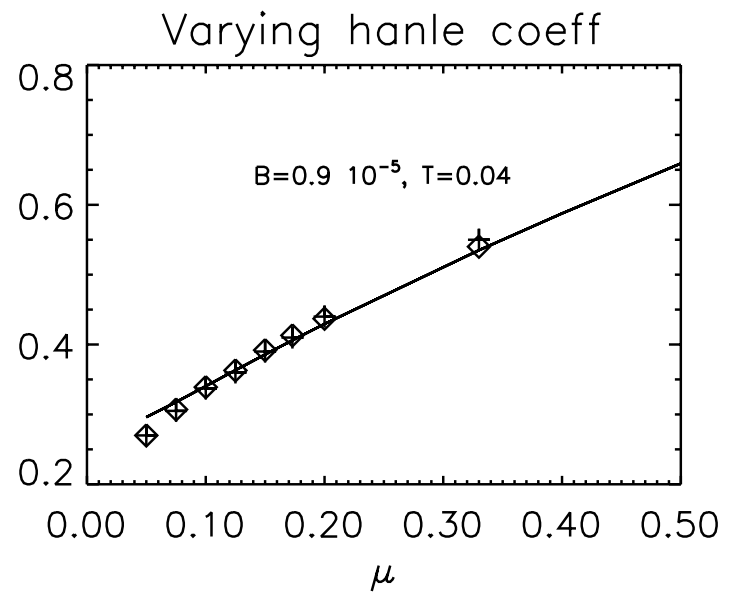

(d)

Fig. 4. Center-to-limb variations of the intensity in lines with $W_{2}=0.4$, for grids of models with a) a set of values for $B_{\text {th }}$ between $0.5 \times 10^{-5}$ and $1.3 \times 10^{-5}$ with a step of $\left.0.1 \times 10^{-5} ; \mathbf{b}\right)$ a set of values of $T$ between 0.02 and 0.14 with a step of $0.02 ; \mathbf{c}$ ) a set of values of $c_{\mathrm{sc}}$ between 0.02 and 0.14 with a step of 0.02 ; d) a set of $W_{B}$ values from 1 . to 0.2 with a step of 0.2 . The symbols show the observed quantities in the $\mathrm{MgH}$ lines.

Table 2. Values of the model parameters for the observed molecular lines.

\begin{tabular}{cccccccc}
\hline \hline Molecular line & Branch $(\mathrm{J})$ & $\Gamma_{\mathrm{R}}\left(\mathrm{s}^{-1}\right)$ & $g_{J}$ & $W_{2}$ & $T$ & $B_{\mathrm{th}}$ & $c_{\mathrm{sc}} W_{B}$ \\
\hline$(\mathrm{MgH}) 515.70 \mathrm{~nm}$ & $\mathrm{Q}(17)$ & $1.78 \times 10^{7}$ & 0.059 & 0.4 & 0.04 & $0.9 \times 10^{-5}$ & 0.05 \\
$\left(\mathrm{C}_{2}\right) 515.76 \mathrm{~nm}$ & $\mathrm{P} 1(30)+\mathrm{P} 2(29)$ & $0.36 \times 10^{7}$ & $\mathrm{P} 1: 0.066, \mathrm{P} 2: 0.003$ & 0.1 & 0.12 & $0.9 \times 10^{-5}$ & 0.12 \\
$\left(\mathrm{C}_{2}\right) 515.77 \mathrm{~nm}$ & $\mathrm{P} 3(28)$ & $0.36 \times 10^{7}$ & -0.065 & 0.1 & 0.06 & $0.9 \times 10^{-5}$ & 0.12 \\
$\left(\mathrm{C}_{2}\right) 515.85 \mathrm{~nm}$ & $\mathrm{P} 1(29)+\mathrm{P} 2(28)$ & $0.36 \times 10^{7}$ & $\mathrm{P} 1: 0.068, \mathrm{P} 2: 0.003$ & 0.1 & 0.12 & $0.9 \times 10^{-5}$ & 0.12 \\
$\left(\mathrm{C}_{2}\right) 515.87 \mathrm{~nm}$ & $\mathrm{P} 3(27)$ & $0.36 \times 10^{7}$ & -0.067 & 0.1 & 0.06 & $0.9 \times 10^{-5}$ & 0.12 \\
$\left(\mathrm{C}_{2}\right) 515.95 \mathrm{~nm}$ & $\mathrm{P} 1(28)+\mathrm{P} 2(27)$ & $0.36 \times 10^{7}$ & $\mathrm{P} 1: 0.071, \mathrm{P} 2: 0.004$ & 0.1 & 0.12 & $0.9 \times 10^{-5}$ & 0.12 \\
$\left(\mathrm{C}_{2}\right) 515.96 \mathrm{~nm}$ & $\mathrm{P} 3(26)$ & $0.36 \times 10^{7}$ & -0.07 & 0.1 & 0.06 & $0.9 \times 10^{-5}$ & 0.12 \\
$(\mathrm{MgH}) 515.98 \mathrm{~nm}$ & $\mathrm{Q}(16)$ & $1.78 \times 10^{7}$ & -0.056 & 0.4 & 0.04 & $0.9 \times 10^{-5}$ & 0.05 \\
$\left(\mathrm{C}_{2}\right) 516.10 \mathrm{~nm}$ & $\mathrm{P} 1(26)+\mathrm{P} 2(25)$ & $0.36 \times 10^{7}$ & $\mathrm{P} 1: 0.076, \mathrm{P} 2: 0.004$ & 0.1 & 0.12 & $0.9 \times 10^{-5}$ & 0.12 \\
\hline
\end{tabular}

Hanle effect of weak unresolved magnetic fields depends only on the absolute value of the Landé factor as shown by Eq. (4).

Let us now see how the ratio of the Stokes parameter $Q$ in both lines depends on the ratio of their Hanle depolarization factors. From Eqs. (12) and (15) we derive

$\frac{Q_{s}(\mu)}{Q_{d}(\mu)}=\frac{S_{Q}^{s}(\mu)\left(1-\exp \left(-T_{s} / \mu\right)\right)+Q_{\mathrm{inc}}(\mu) \exp \left(-T_{s} / \mu\right)}{S_{Q}^{d}(\mu)\left(1-\exp \left(-T_{d} / \mu\right)\right)+Q_{\mathrm{inc}}(\mu) \exp \left(-T_{d} / \mu\right)}$, where we denote by the indexes $s$ and $d$ the quantities referring to the unblended and blended lines respectively. As the polarization in the lines is much larger than in the continuum the second term in the expressions of $Q$ may be neglected in a first approximation. Expanding the exponential term for small values of the optical depths $T_{s}$ and $T_{d}$ we write

$\frac{Q_{s}(\mu)}{Q_{d}(\mu)} \simeq \frac{S_{Q}^{s}(\mu) T_{s}}{S_{Q}^{d}(\mu) T_{d}}$ 

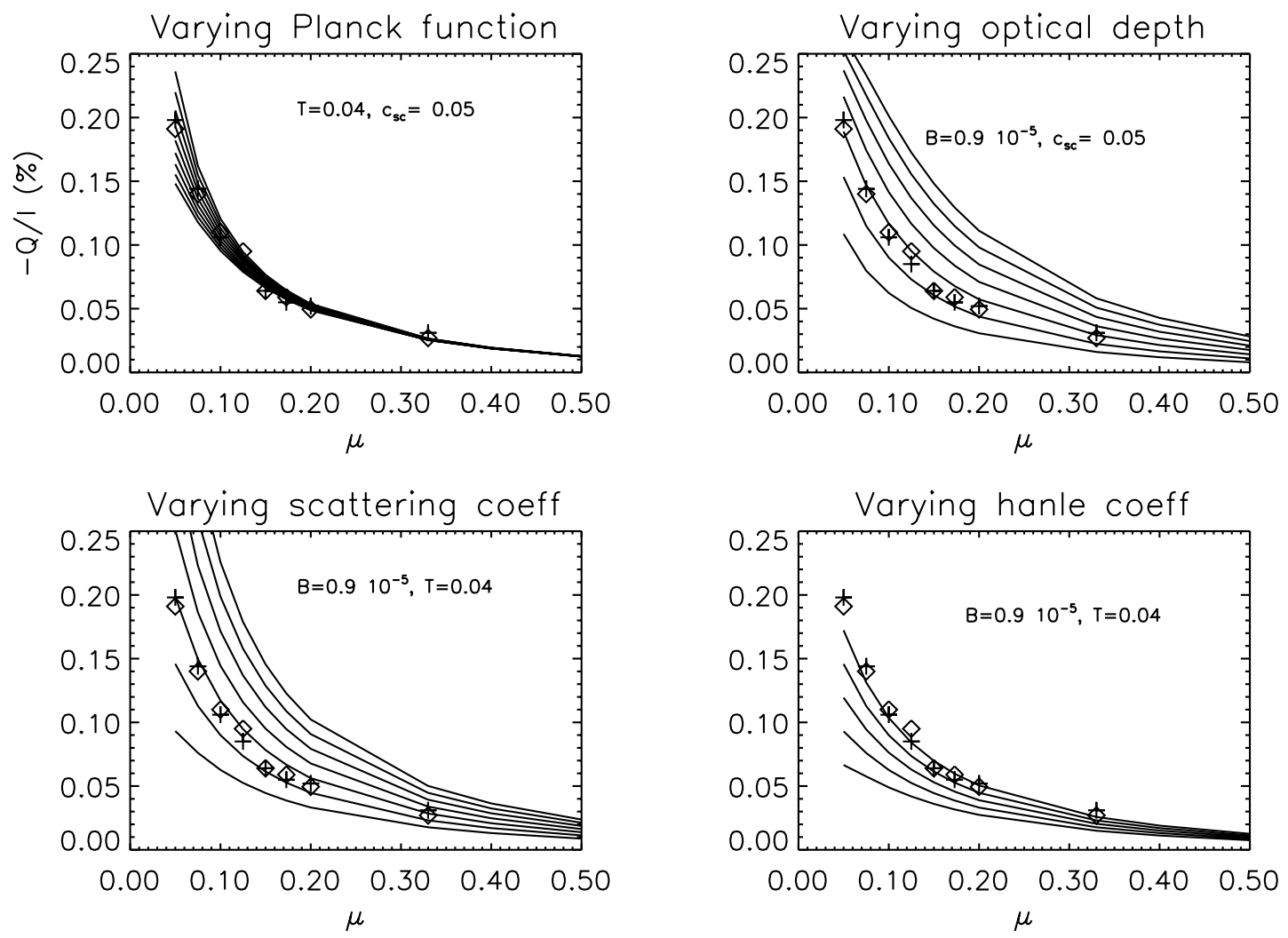

Fig. 5. Center-to-limb variations of the polarization in lines with $W_{2}=0.4$, for the same parameter values as in Fig. 4 .
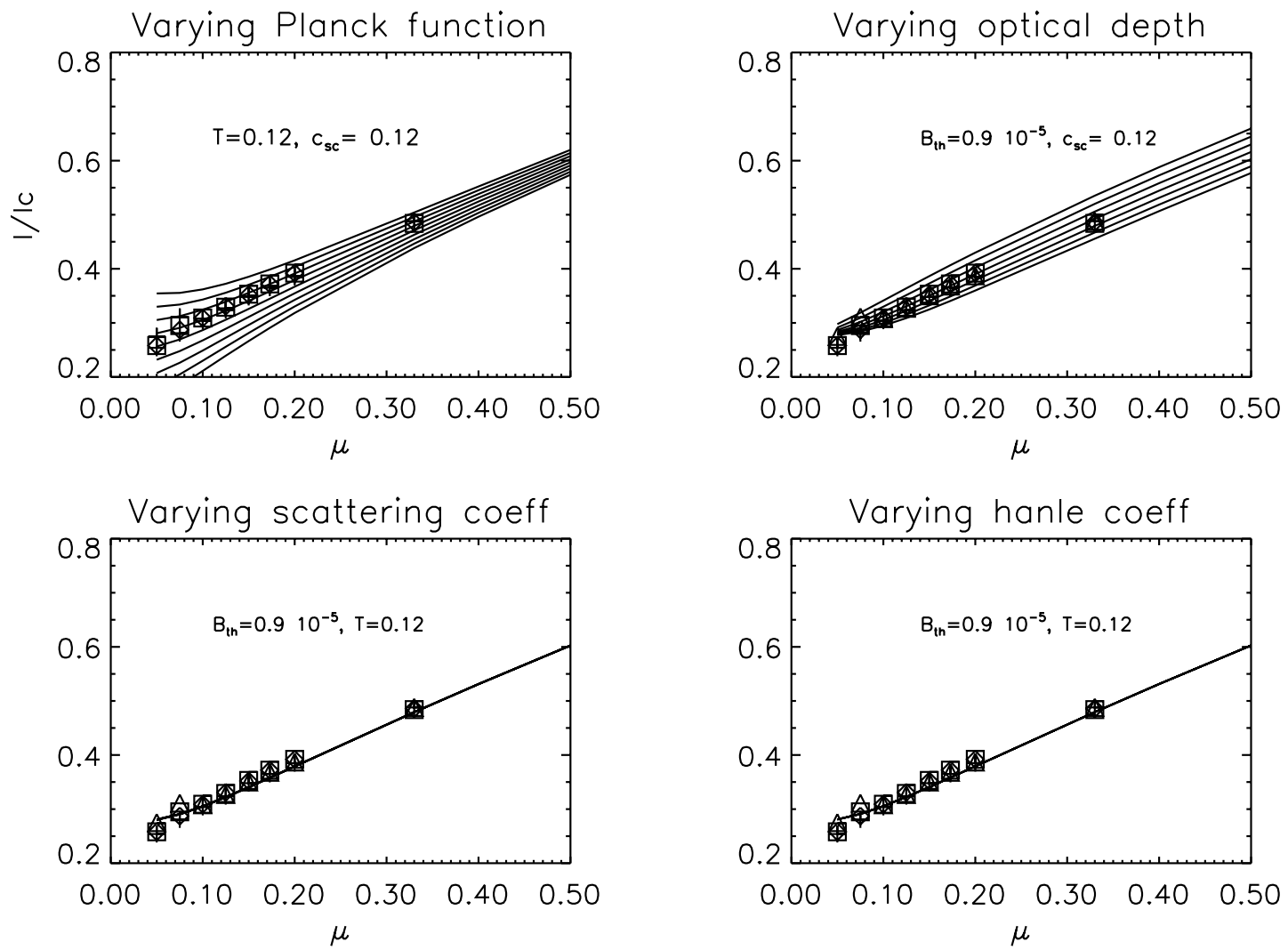

Fig. 6. Same as Fig. 4, for lines with $W_{2}=0.1$. The symbols show the observed quantities in the $\mathrm{C}_{2}$ blended lines. In $\mathbf{b}$ ), the set of values of $T$ ranges from 0.04 to 0.16 with a step of 0.02 . 

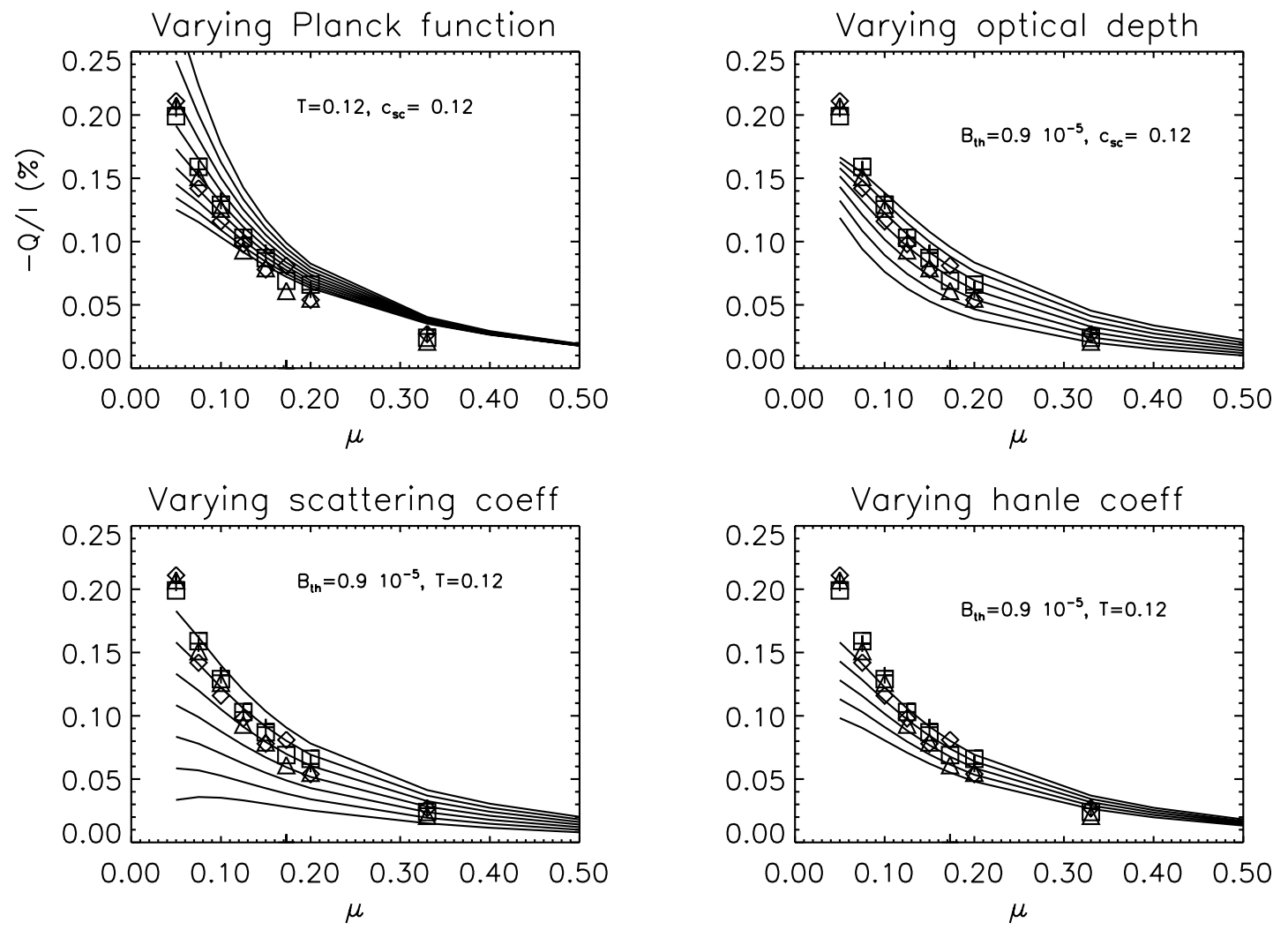

Fig. 7. Same as Fig. 5 for lines with $W_{2}=0.1$ and for the $C_{2}$ blended lines. b) shows the same set of values for $T$ as Fig. 6 b.
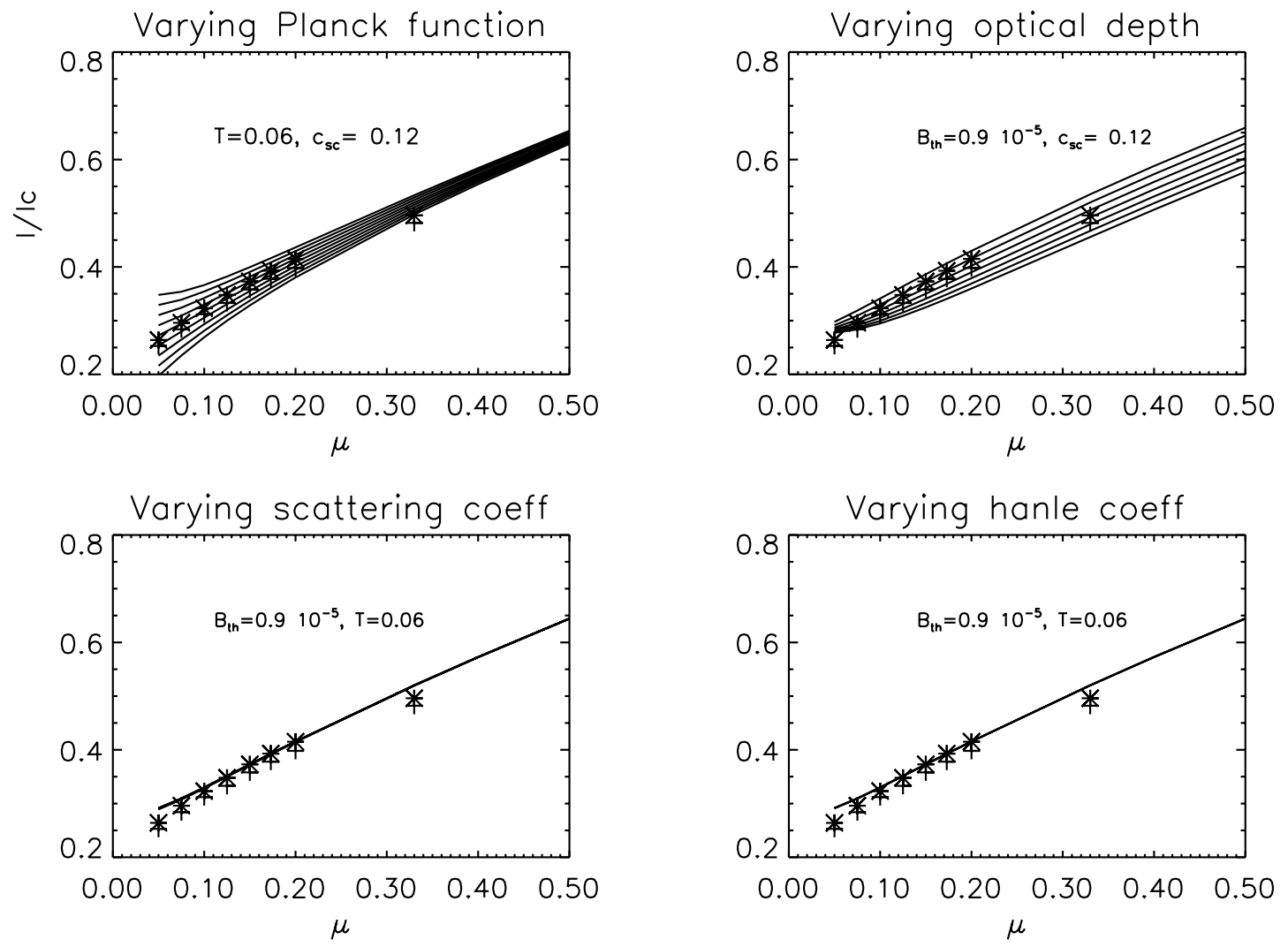

Fig. 8. Same as Fig. 6, for the $C_{2}$ unblended lines. 

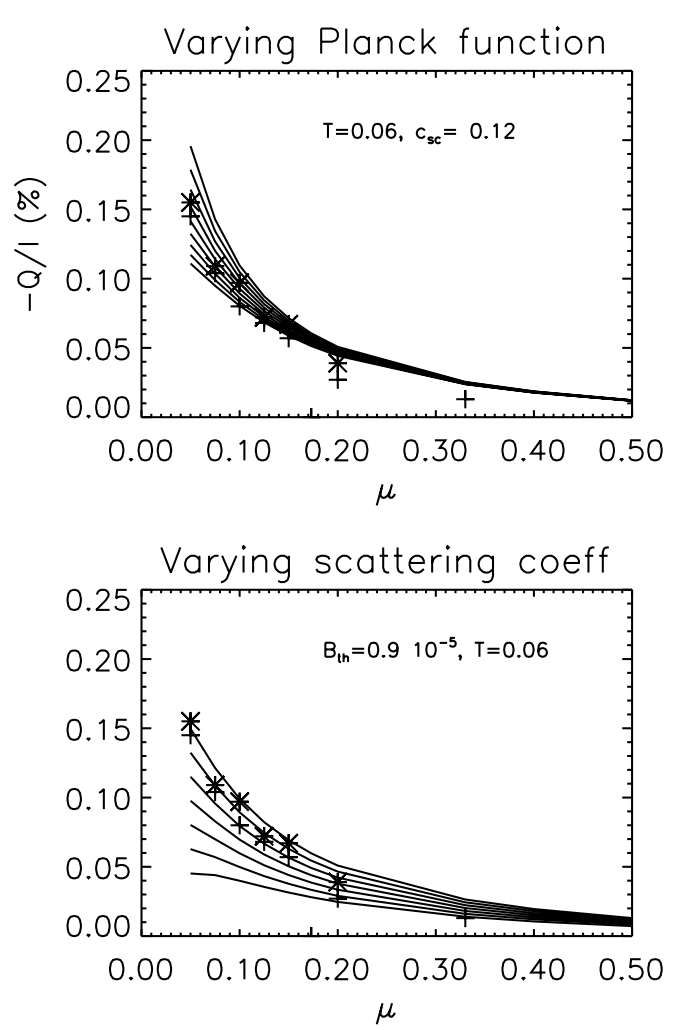

Fig. 9. Same as Fig. 7 for the $C_{2}$ unblended lines.

The source functions $S_{Q}^{s}(\mu)$ and $S_{Q}^{d}(\mu)$ depend on the product $c_{s c} W_{B}$ and on an integral term depending on the anisotropy and polarization of the continuum and on the line optical depth. Using the fact that the scattering coefficients are identical for blended and unblended lines and introducing the values of the line optical depths we derive

$\frac{Q_{s}(\mu)}{Q_{d}(\mu)} \simeq 0.5 \frac{W_{B}(s)}{W_{B}(d)} \frac{I_{1}\left(T_{s}\right)}{I_{1}\left(T_{d}\right)}$

where $I_{1}\left(T_{s, d}\right)$ are given by Eq. (16).

We notice that the ratio $I_{1}\left(T_{s}\right) / I_{1}\left(T_{d}\right)$ is different from unity because it depends on the optical depths of the blended and unblended lines. The line optical depths have to be known, together with the continuum radiation anisotropy and polarization, in order to derive the ratio of the Hanle depolarization factors from the observed values of the Stokes parameter $Q$ in both types of lines. The observed values of $Q_{s}(\mu) / Q_{d}(\mu)$ have been obtained by averaging over the blended and unblended lines respectively.

Figure 10 shows how this ratio varies as a function of $\mu$. The different curves corresponding to different values of $W_{B}(s) / W_{B}(d)$ are derived from the full modelling and not from the approximate expression (18). Comparing with the observed $Q$ ratio we can determine $W_{B}(s) / W_{B}(d)$, its value is between 0.8 and 1 . for $\mu \leq 0.2$, and decreases to 0.62 at $\mu=0.33$. The ratio $W_{B}(s) / W_{B}(d)$ is related to $\gamma_{\mathrm{H}}(s)$ and $\gamma_{\mathrm{H}}(d)$ as shown by Eq. (4). As the blended and unblended lines have the same oscillator strength and probably the same depolarizing collision rates, we can write

$\gamma_{\mathrm{H}}(s) / \gamma_{\mathrm{H}}(d)=g_{\mathrm{eff}}(s) / g_{\mathrm{eff}}(d)=2$
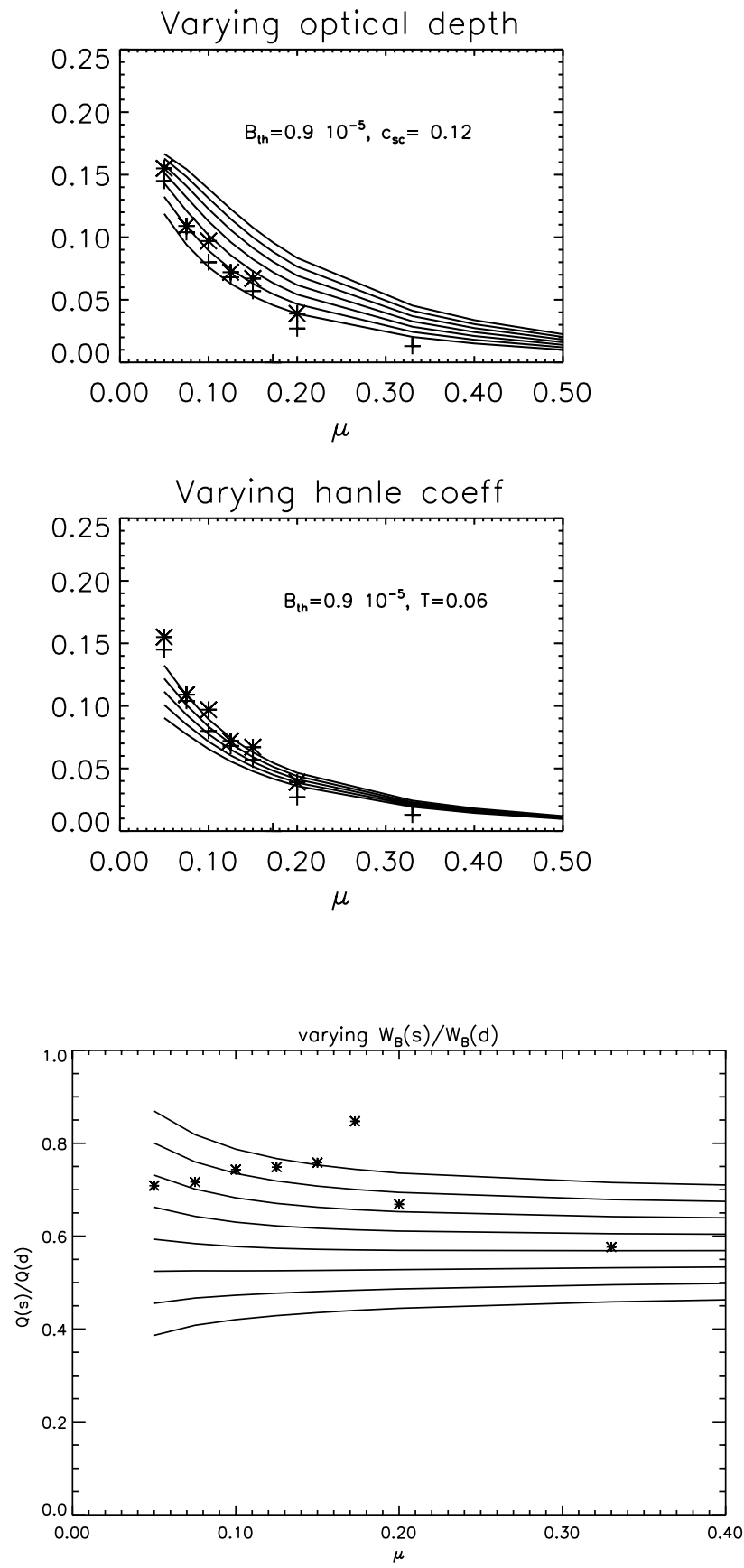

Fig. 10. Center-to-limb variations of the ratio of the Stokes parameter $Q$ in unblended and blended lines of $\mathrm{C}_{2}$ for different values of the ratio $W_{B}(s) / W_{B}(d)$. From the top to the bottom the different curves correspond to decreasing values of $W_{B}(s) / W_{B}(d)$ from 1 . to 0.3 , by step of 0.1 . The symbols refer to the observed values.

Using this equality and the values of $W_{B}(s) / W_{B}(d)$ derived above, we can determine $\gamma_{\mathrm{H}}(s)$ and $\gamma_{\mathrm{H}}(d)$. We find that $\gamma_{\mathrm{H}}(s)$ is between 0 . and 0.5 for $\mu \leq 0.2$, and increases to 1.6 at $\mu=0.33$. This last value is not precisely determined because of the saturation of the Hanle depolarization factor for large $\gamma_{\mathrm{H}}$. The corresponding strength of the unresolved magnetic field is smaller or on the order of 15 Gauss for $\mu \leq 0.2$ and increases to values on the order of 45 Gauss at $\mu=0.33$. 
Such a weak magnetic field gives $W_{B}(\mathrm{MgH}) \simeq 1$ for $\mu \leq 0.2$ and $W_{B}(\mathrm{MgH}) \simeq 0.88$ at $\mu=0.33$, it will thus only weakly affect the polarization of the $\mathrm{MgH}$ lines.

Let us stress here that the determination of $W_{B}$ from the observations of the line polarization requires to model accurately enough the coupling between the line and continuum radiation field. In particular we found that the polarized line source function given by Eq. (15) depends on the polarization and limbdarkening of the continuum and on the line optical depth.

\section{Discussion and conclusion}

The diagnostics procedure described in this paper relies of the differential Hanle effect in $\mathrm{C}_{2}$ lines, together with a detailed modeling of the line formation process. Differential Hanle effect is likely to depend very weakly on the presence of depolarizing collisions because they would affect all the $\mathrm{C}_{2}$ lines in the same way. It thus provides quite a powerful diagnostic tool.

In Paper I we presented observations performed in the same spectral domain above the solar limb. In this case the scattering of the photospheric radiation by the molecular transitions gives rise to polarized emission lines. They give access to the diagnostics of the temperature minimum region between the photosphere and the chromosphere. We used a simple single-scattering model to interprete these observations and we showed that in the absence of Hanle effect the line polarization rate depends on the intrinsic polarizability $W_{2}$ and on the continuum photospheric radiation. This yields results in agreement with the theory for the $\mathrm{MgH}$ lines, but in excess by a factor of about two for the $\mathrm{C}_{2}$ lines. This discrepancy is reduced when one takes into account the polarization of the incident continuum radiation, which was neglected in Paper I, however an excess of polarization still remains in the $\mathrm{C}_{2}$ lines, with respect to the expected non magnetic scattering polarization.

We suspect that this problem may be due to the reduction procedure that was used to remove the stray light from the observed spectra. If we examine the intensity and polarization ratio of the unblended and blended lines of $\mathrm{C}_{2}$, derived from the observations shown in Figs. 1 and 2 of Paper I, we obtain $I_{s} / I_{d} \simeq 0.62$ and $Q_{s} / Q_{d} \simeq 0.63$. But the values that we expect for optically thin scattering lines in the absence of a magnetic field are $I_{s} / I_{d}=0.5$ and $Q_{s} / Q_{d}=0.5$. This discrepancy indicates that the reduction procedure which was used to correct the spectrum from stray light has lead to an overestimation of the polarization in the $\mathrm{C}_{2}$ lines. This has to be investigated further. Reliable measurements of the polarization of these scattering lines formed in the low chromosphere would give access to the determination of weak magnetic fields in this layer by applying the procedure that we have described in the present paper.
Acknowledgements. We thank the THEMIS technical staff and the support astronomer F. Paletou for their help during the observations and J.O. Stenflo and S. V. Berduygina for valuable comments.

THEMIS is operated on the Island of Tenerife by CNRS-CNR in the Spanish Observatorio del Teide of the Instituto de Astrofísica de Canarias.

\section{References}

Arnaud, J., Mein, P., \& Rayrole, J. 1998, in a Crossroads For European Solar and Heliographic Physics, Tenerife, March 1998, ed. ESA SSPG and IAC, ESA SP-417, 213

Arnaud, J., Faurobert, M., Vigneau, J., \& Paletou, F. 2001, in Advanced Solar Polarimetry - Theory, Observation and Instrumentation, ed. M. Sigwarth, ASP Conf. Ser., 236, 151

Berdyugina, S. V., Stenflo, J. O., \& Gandorfer, A. 2002, A\&A, 388, 1062

Berdyugina, S. V. 2003, private communication

Chandrasekhar, S. 1960, Radiative Transfer (New York, Dover)

Donati, J.-F., Semel, M., Rees, D. E, Taylor, K., \& Robinson, R. D. 1990, A\&A, 232, L1

Faurobert, M., Arnaud, J., Vigneau, J., \& Frisch, H. 2001, A\&A, 378, 627

Faurobert, M., \& Arnaud, J. 2002, A\&A, 382, L17

Fontenla, J. M., Avrett, E. H, \& Loeser, R. 1993, ApJ, 406, 319

Gandorfer, A. 2000, The Second Solar Spectrum, a high spectral resolution polarimetric survey of scattering polarization at the solar limb in graphical representation (vdf Hochschulverlag AG and der ETH Zürich), ISBN 3728127647

Keller, C. U., Sheeley, JR. N. R. 1999, in Solar Polarization, ed. K.N. Nagendra, \& J.O. Stenflo (Kluwer), 17

Leroy, J. L. 1972, A\&A, 19, 287

Mickey, D. L., \& Orall, F. Q. 1974, A\&A, 31, 179

Mohan Rao, D., \& Rangarajan, K. E. 1999, ApJ, 524, L139

Paletou, F., \& Molodij, G. 2001, in Advanced Solar Polarimetry Theory, Observation and Instrumentation, ed. M. Sigwarth, ASP Conf. Ser., 236, 9

Paletou, F., Lopez-Ariste, A., Bommier, V., \& Semel, M. 2001, A\&A, 375, L39

Pierce, A. 1968, ApJS, 17, 1

Semel, M. 1994, in Solar Surface Magnetism, ed. R.J. Rutten, \& C.J. Schrijver (Kluwer)

Semel, M. 2003, A\&A, 401, 1

Solanki, S. K., Livingston, W., Muglach, K., \& Wallace, L. 1996, A\&A, 315, 303

Stein, R., \& Nordlund, A. 1989, ApJ, 342, L95

Stenflo, J. O. 1982, Sol. Phys. 80, 209

Stenflo, J. O. 1994, Solar Magnetic fields (Dordrecht: Kluwer)

Stenflo, J. O., \& Keller, C. 1997, A\&A, 321, 927

Stenflo, J. O., Bianda, M., Keller, C. U., \& Solanki, S. K. 1997, A\&A, 322,994

Stenflo, J. O., Keller, C. U., \& Gandorfer, A. 1998, A\&A, 329, 319

Wiehr, E. 1975, A\&A, 38, 303

Wiehr, E., \& Bianda, M. 2003, A\&A, 398, 739 